\title{
Study of family medicine role in breast cancer prevention: A systematic review
}

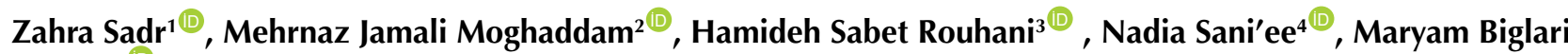 \\ Abhari $^{3^{*}}$ \\ 'Department of Community and Family Medicine, Firooz Abadi Hoapital, Iran university of Medical Sciences, Tehran, Iran \\ ${ }^{2}$ Family Medicine Specialist, Iran university of Medical Sciences, Tehran, Iran \\ ${ }^{3}$ Preventive Medicine and Public Health Research Center, Psychosocial Health Research institute, Community and Family \\ Medicine Department, School of Medicine, Iran University of Medical Sciences, Tehran, Iran \\ ${ }^{4}$ Department of Medical Library and Information sciences, Iran University of Medical Sciences, Tehran, Iran
}

\section{Correspondence to:}

Maryam Biglari Abhari, Email;

Biglari.m@iums.ac.ir

Received: 25 Apr. 2021

Accepted: 3 Aug. 2021

ePublished: 15 Aug. 2021

Keywords: Family physician, Breast cancer, Prevention

\begin{abstract}
Breast cancer is one of the most common cancers and is one of the biggest health threats in women around the world. Since the systematic review study in Iran has not been conducted so far, this study was designed to determine the role of family physicians or first-level care physicians in preventing breast cancer at various levels. In this systematic review, we found family physicians could play a significant role in all levels of breast cancer prevention, including roles in education, risk assessment and early detection of cancer, treatment and follow-up of patients with breast cancer and rehabilitation, and help improve quality of life. Survivors and those treated for breast cancer.
\end{abstract}

\section{Introduction}

Despite progress in controlling and preventing communicable diseases, the incidence and prevalence of noncommunicable diseases has increased dramatically over the past decades (1). In many developed and developing countries, cancer is considered as one of the most important health problems (2). In some countries, cancer is the second leading cause of death after cardiovascular disease. It is the third cause of death after cardiovascular diseases and injuries in Iran (3). Breast cancer accounts for about one third of all cancers in women, the second prevalent and cancer after lung cancer and the leading cause of cancer death among women $(1,4,5)$. According to global statistics, the annual incidence of this cancer is increasing rapidly specially in countries with lower in past $(6,7)$. Each year, over 1150000 new cases and 502,000 deaths of breast cancer in women around the world (8) The risk of developing this cancer during the life course of a woman is 1 to 8 , and seems to be rising (1).

The incidence of breast cancer is increasing In Iran and it is often detected in high stages. The age of onset is almost 10 years less than Western countries $(9,10)$.

About $85 \%$ of women with breast cancer

\section{Key point}

Family physicians at all levels of breast cancer prevention can play a significant role in educational, risk assessment and early diagnosis of breast cancer.

have a negative familial history (1) survival depends on the size of the tumor more than its type. Given the high prevalence of breast cancer and the incidence and mortality throughout the world, it is necessary to focus on its prevention. Half of deaths related to cancers could be prevented by healthy lifestyle, risk assessment and routine evidence-based screening and more effective treatment $(11,12)$. One-third of cases can be cured completely if only they are detected early and treated on time (13).

By regular screening through mammography and annual breast physical exam by the physician, the death rate due to breast cancer has been reduced in recent years $(14,15)$. Family physicians play an essential role in the prevention, diagnosis and management of breast cancer (1).

Since family doctors often have a longterm relationship with patients, they trust them to implement recommendations (1). In the United States, family physicians play the

Copyright $(0) 2021$ The Author(s); Published by Society of Diabetic Nephropathy Prevention. This is an open-access article distributed under the terms of the Creative Commons Attribution License (http://creativecommons.org/licenses/by/4.0), which permits unrestricted use, distribution, and reproduction in any medium, provided the original work is properly cited. 
role of gatekeepers for health care. Therefore, patients are more visited by family physicians than other specialists (1). Family physicians should be able to do breast examination to detect anomalies and teaching their patients for selftesting of breast at the same time (1).

Almost, evaluation of breast masses and assessment of risk is started with family history of breast cancer. Family physicians should identify risk factors which include modifiable factors such as; first pregnancy after 35 years of age, non-lactation, alcohol and tobacco use, hormone therapy and also menopausal age above 55 years, menstrual age before the age of 12 , positive genetic and familial history, history of endometrial, ovarian or colon cancers $(1,16)$.

\section{Objectives}

Considering that family physicians have the first level of dealing with the patient and have the highest number of referrals, they can act effectively in modifiable risk factor control. On time, diagnostic approaches are critical to reduce burden of disease by effective intervention. Since no systematic review has been conducted so far, this study was designed to determine the role of family physicians in preventing breast cancer at all levels of prevention through a systematic review.

\section{Methods}

This is a systematic review conducted at Iran university of medical sciences, faculty of medicine, department of community medicine and family medicine, from June 2018 to February 2019.

Inclusion criteria contain; articles in Persian and English languages, with available full text in accessed databases published in the years of 2000 to 2019.

Exit criteria include; articles in other languages with no accessibility to their full text and those not indexed in available databases.

\section{Search strategy}

Initially, the structured study question (PICO) was developed as $\mathrm{P}$; breast cancer and $\mathrm{O}$; increased survival and promotion of women's health (the absence of breast cancer or completed treatment and the survival of those who were affected). Specific research questions were also compiled what is the educational role of family physicians in preventing breast cancer? In all levels of prevention, what is the role of family physicians in diagnosis and screening of breast cancer? In secondary prevention, what is the role of family physicians in breast cancer treatment process? What is the role of family physicians for rehabilitation in breast cancer? (tertiary prevention).

The searching process was performed independently by three researchers, after selecting appropriate keywords through the $\mathrm{MeSH}$ and searching for similar articles and finding other related keywords. The resulting keywords included; 1. Family physician OR family doctor OR general practitioner OR primary care physician, 2. Role OR duty OR action OR performance, 3. Screening OR risk assessment OR early detection OR prevention OR diagnosis OR care OR treatment OR rehabilitation OR palliative OR supportive, 4. Breast cancer OR breast malignancy OR breast malignant tumor OR breast malignant mass

To collect the related evidence, we searched the Web of Knowledge, PubMed, Scopus and also family medicine electronic journals not indexed in these databases. Iranian databases containing Irandoc, SID, ISC, Magiran, were reviewed as well as family medicine curriculums. The search strategy for each database is presented in online Supplementary file 1 . No filter was applied to increase the search sensitivity except for the years of publication (2000 to 2019).

\section{Data collection}

After searching, all retrieved articles were imported into EndNote 7 software to go through the next steps. The studies were first reviewed on the basis of matching titles with keywords and research questions by three independent researchers. Then, the matched articles were entered into the next stage. The case of disagreement between three researchers was solved by fourth researcher in a panel. Then, these items were reviewed in terms of abstract content in accordance to research questions and purposes and related items entered the next stage. Now, the full text of articles was reviewed and disagreement was followed and solved by the presence of fourth researcher. Articles fully matched with research questions and objectives were offered to two independent experts for critical appraisal. After gaining scores more than $75 \%$ in critical evaluation of articles, they were entered the final stage of review and extraction of the main results of the research. The full details of selected articles are reported in Supplementary file 2. After reviewing the final included articles, their references were also evaluated. Out of all 614 references of 13 articles were matched with keywords, questions and research objectives (in terms of title), and finally of these 13 papers, six were eligible to enter the final stage of study. A total of 19 articles were imported into the results table. These articles attained critical scores over $75 \%$ and entered the final step to extract the main findings.

\section{Critical appraisal}

To appraise the articles, Critical Appraisal Skills Program (CASP) evaluation checklist (17) was used, which is valid and contains special checklists for each type of study. For cross-sectional studies, the STROBE (18) checklist was used for review and scoring. Each item with checklist criteria was accepted and scores 1 otherwise, 0 points. The final score is calculated as follows:

Final scor $(\%)=\left(\frac{\text { number of accepted items }}{\text { total number of items }}\right) * 100$ 


\section{Results}

We achieved 4469 articles through searching in databases and 684 articles in three journals of family medicine. Hence, a total of 5153 articles, were obtained. However, there was no relevant evidence in search of Iranian databases and family medicine curriculums. After removing 244 duplicates, 4908 articles remained. These articles were assessed in terms of matching the title with research questions and objectives. In this stage, 4637 articles were excluded and 272 articles were included in the next stage. In terms of matching the abstract content with the research questions and objectives, 200 articles were excluded from this study. Around 72 articles were entered into the next stage and the full text of articles was reviewed for adaptation to the research. The final result was exclusion of 59 articles and the entrance of 14 papers to the next stage, namely, critical evaluation. The steps are outlined in Figure 1 based on PRISMA policy.

Out of 14 remaining papers, there were two qualitative studies, five cross-sectional surveys, two reviews, two systematic reviews, two interventional study papers and one expert opinion.

The scores obtained in appraisal were $53 \%$ to $92 \%$, the highest score was for a qualitative and an interventional study, and the least was the expert opinion. Articles scored over $75 \%$ were included in the study, and articles with scores lower than $75 \%$ were excluded. Finally, 13 papers remained for the final data extraction, along with six articles from references, in a total of 19 articles. Scores obtained in critique-extracted data of each article are described in detail in online Supplementary file 2. Then we categorize the findings based on research goals and questions which is presented in online Supplementary file 3.

\section{Discussion}

In order to develop effective and scientific roles for family physicians in the field of breast cancer prevention at all levels, interventions should be designed natively and tailored to the views of the patients themselves, family physicians and the cancer care team as well as evidences and guidelines. Considering the role of family physicians in first-level prevention is of paramount importance, and

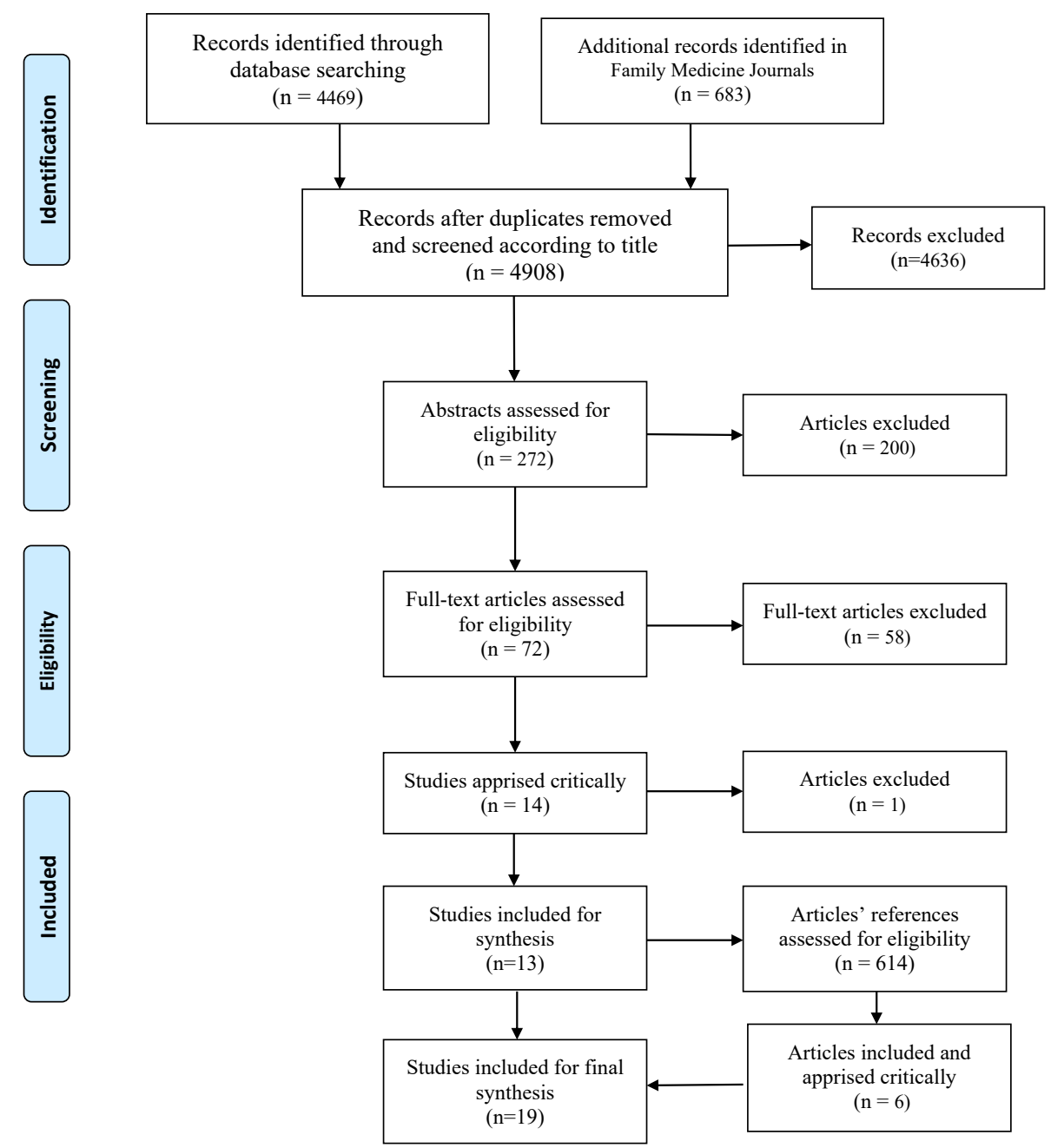

Figure 1. PRISMA flow diagram of the study. 
studies should be designed to address this issue.

\section{Educational role}

In our study, four articles refer to the role of family doctor, with only two related to first-level prevention and only moderated lifestyle. Women's awareness of breast cancer and breast self-examination play a very important role in prevention of breast cancer. Nevertheless, few studies have addressed the role of family physician training, especially in first-level prevention. Educating the family physician as a first-line prevention can be complete and comprehensive and will raise awareness of all aspects of breast cancer, thereby enhancing the prevention of breast cancer at all levels.

Montazeri et al, in a study, examined the awareness of breast cancer and breast self-examination in 1402 women in Tehran. About $85 \%$ of them were married and $94 \%$ had no history of masturbation problems, and $5 \%$ had a history of benign mammary problems and $1 \%$ had a history of malignant mumps. Around $64 \%$ of respondents were familiar with breast cancer, $61 \%$ believed that the disease was relatively common among Iranian women. Most women (44\%) knew a painless mass as breast cancer, $37 \%$ reported having breast self-examination; however, only $17 \%$ said they were performing regular examinations and the reason for the rest of the women who did not conduct the examination was to say they did not know how to do it. This study showed that women's familiarity with breast cancer symptoms is low and their use of effective screening methods, like clinical examination and mammograms, is inadequate and concluded that primary care physicians should focus on increasing women's awareness through effectively educating and encouraging them to visit and see as soon as possible any symptoms (8). Previously, the study by Chukmaitov et al, on 500 women in Almaty, Kazakhstan, showed that counseling for doctors is effective in preventing breast cancer. They concluded that doctors should include women in their coverage to participate in teaching and promoting screening programs (19). Given the importance of educational roles, it seems that special attention has to be paid to this, and interventions are designed to develop educational methods and curricula based on first-level prevention and assessment of their effectiveness. Some kind of training is part of the duties of a family doctor.

The 2008 study by Zheng et al, found that half of cancerrelated deaths were reduced by better screening services, which is more effective than treatment. Death depends on which stage of cancer is diagnosed as the best prognosis for those cases that are diagnosed early. Of the common barriers in the level of primary care and professional care, there is a lack of sufficient knowledge about the risk assessment of cancer. In addition, public acceptance in different societies varies with the risk factors of cancer and exposure to them (20).

Moreover, Amir et al showed that breast cancer prevention strategies are most widely used in women at high risk for cancer, which can potentially increase the incidence of breast cancer to 1500 cases per 100000 cases, while among low-risk women, this is, at best, 25 per 100000 cases. As a result, it is imperative that a precise risk assessment is conducted individually in order to select the right women for prevention programs (21). Hauk suggested that breast cancer screening should be conducted in women aged 40 years and older and in women aged 30 to 30 every 1-3 years. Studies support clinical examination with mammograms. Although most women with breast cancer do not have identifiable risk factors, specific characteristics increase the risk of this cancer compared to the normal population. Physicians should regularly evaluate these risk factors (22). A study by Chang and Brown, suggested that family physicians play a key role in identifying patients who need more care because of a family history of breast cancer (23).

Genetic tests do not always provide enough information, but positive family history can help to screening. American College of Medical Genetics and Genomics (ACMG) and the American Academy of Pediatrics (AAP) recommends that genetic testing in asymptomatic children with a low risk for cancer does not go away. Unless these interventions reduce morbidity and mortality (23).

The main components of cancer risk assessment include; information on the history of cancer in a person or relatives, identifying people at risk for cancer, screening for cancer in people at high risk, referring high risk individuals to cancer genetic clinics and rarely a family history of a hereditary cancer syndrome, which requires referral to genetic clinics for cancer (17).

Additionally, Kaplan et al, in their study, referred to the importance of referrals for screening and evaluations (24). Furthermore, Poole and colleagues conducted a study on 15,195 Canadian women aged 50-69 years, indicating that women under the active care of family physicians received more mammography screening and concluded that women treated with family doctors are more likely to participate in screening and mammograms (25).

The study by Avery and Daniel showed that family physicians in the prevention, diagnosis and management of cancer breast play an important role and evaluation of breast masses begins with a family history of breast cancer. Examinations that are practicable by the family physician include; examination of the masses, examination of areas suspected by the patient, touching the lymph nodes of the areas of axilla and supraclavicular area (1).

The present study seems to respect almost all aspects of this role, including the importance of hereditary cases and the assessment of the genetic risk of cancer; however, there was no clear mention of periodic breast examinations. Therefore, the need for a specific examination with a greater focus on these cases is felt. However, in other aspects, such as genetic evaluations and the screening of other diseases and cancers, such as the normal population, 
its attention and importance are expressed.

It should be taken into account in almost all aspects. Focus on controlling physical symptoms, especially pain that improves the patient's quality of life and helps accelerate his return to day-to-day activities.

The control of the complications of the disease itself and the complications of treatment by the family doctor is confirmed.

The necessity of referring the patient to higher levels also emphasizes the need for attention to psychosocial support and treatment and the importance of proper follow-up of the patient in terms of adherence to treatment and his support during the treatment route. Grunfeld et al conducted a randomized clinical trial comparing the follow-up of breast cancer patients by family physicians and specialized cancer clinics in terms of initial outcomes (recurrence) and secondary outcomes (quality of life). This study has been conducted from 1997 to 2001, 968 women with breast cancer, whose initial treatment was complete and with a history of illness, was 9 to 15 months old, with a diagnosis of the disease. About 483 patients were in the family physicians group and 485 in the cancer clinic group followed by 3.5 years. In the group of family physicians, there were 54 cases of relapse (11.2\%) and 29 cases of death $(0.6 \%)$. Moreover, there were 64 cases of relapse (13.2\%) and 30 deaths (6.2\%), with no significant difference between the two groups. Therefore, the follow up by the family physician would be reasonable and safe (26).

Due to an increase in breast cancer cases and insufficient number of oncologists, Maly et al, found that a number of women are deprived of specialized care services. The participation of first-level physicians in low-income medical care, due to lower costs and easier access compared to specialized cancer centers, has had better results in tracking breast cancer. Therefore, more role should be given to these doctors in the care of breast cancer patients (27).

\section{The role of rehabilitation}

According to the National Cancer Act of 1971 and the National Cancer Rehabilitation Plan in 1972, rehabilitation on cancer has four goals; 1. Psychosocial support, 2. Optimizing physical performance, 3. Optimizing social performance, 4 . Professional advice.

This rehabilitation is done in a variety of ways with an interdisciplinary approach by a physician, oncologist, social worker, psychiatrist, physiotherapist, occupational therapist, nutritionist and others. Rehabilitation interventions can be both preventive and in order to restore performance or supportiveness and relief (28). Therefore, the importance of rehabilitation in cancer patients, including patients with breast cancer, cannot be ruled out.

In spite of the importance of this case, due to the increase in the number of breast cancer treatments and the longevity of the patients, there was not enough attention to this area in the evidence. In our study, only one article (29) showed that cardiovascular rehabilitation through the training of physical activity has been emphasized in the guidelines; however, in other aspects of rehab, the important details that can be provided and managed by the family physician are not addressed. What has been emphasized both by evidence and by doctors and patients (in four articles) is mental support, which is absolutely necessary and necessary, however not enough.

\section{The role of family physicians in palliative care}

Relief-supportive care may be implemented as a sequence of rehabilitation (if it begins with the diagnosis and treatment of cancer) or as a separate part in cancer patients. The purpose of this part of the care in the early stages of the disease (support) is to help better control the physical and psychological symptoms of the patient and help him decide (29).

However, in the final stages, this care is a relief that helps to improve the quality of life in the patient (30) and also avoids imposing unnecessary and harmful therapeutic interventions. The positive effects of providing this part of the service in reducing costs the additional and unproductive burden imposed upon the patient and the health system should not be ignored (31). Evidence from this is not enough to address the possible roles of family physicians, which are part of the fourth level of prevention. A reference to the role of family physicians in palliative care was observed in two articles in general.

\section{Suggestions}

It seems that, we need to study to assess the status of ourselves to determine the level of knowledge and ability of family physicians and the awareness of patients in treating breast cancer and to determine the status of existing protocols in the country for efficiency and up-todate and find out the process of dealing with breast cancer.

\section{Conclusion}

Family physicians at all levels of breast cancer prevention can play a significant role in educational, risk assessment and early diagnosis of cancer, collaborating with a specialized team in the treatment and follow up of patients with breast cancer and rehabilitation and help improve the quality of life of survivors and recipients of breast cancer.

\section{Authors' contribution}

ZS, MJM and HSR conducted the searches. MBA conducted the analysis. Primary draft by NS and MBM. ZS conducted a revision. All authors read and signed the final paper.

Conflicts of interest

The authors declare that they have no competing interests.

Ethical issues

In search of and evidence of evidence, all ethical research points were observed and for studying, the ethics code was received 
from the Ethics Committee of the Vice-Chancellor of Research of Iran University of Medical Sciences (Ethical code \# IR.IUMS.FMD. REC.1397.253). Accordingly, ethical issues (including plagiarism, data fabrication, double publication) have been completely observed by the authors.

\section{Funding/Support}

None declared.

\section{Supplementary Materials}

Supplementary file 1 . The search strategy of study.

Supplementary file 2. Final reviewed articles and extracted data.

Supplementary file 3. Extracted roles for family physician in breast cancer prevention through reviewed articles.

\section{References}

1. Avery D, Daniel M, A family physician's role in the prevention, diagnosis, and management of breast cancer. Am J Clin Med. 2010: 7;76-79.

2. Cabanes A, Vidal E, Aragonés N, Pérez-Gómez B, Pollán M, Lope V, López-A bente G. Cancer mortality trends in Spain: 1980-2007. Ann Oncol. 2010;21 Suppl 3:iii14-20. doi: 10.1093/annonc/mdq089.

3. Mousavi SM, Gouya MM, Ramazani R, Davanlou M, Hajsadeghi N, Seddighi Z. Cancer incidence and mortality in Iran. Ann Oncol. 2009;20:556-63. doi: 10.1093/annonc/ mdn642.

4. Etemadi A, Sadjadi A, Semnani S, Nouraie SM, Khademi H, Bahadori M. Cancer registry in Iran: a brief overview. Arch Iran Med. 2008;11:577-80.

5. Burkman RT. Berek \& Novak's gynecology. JAMA. 2012;308:516-7. doi: 10.1001/jama.308.5.516

6. Wilson CM, Tobin S, Young RC. The exploding worldwide cancer burden: the impact of cancer on women. Int J Gynecol Cancer. 2004;14:1-11. doi: 10.1111/j.1048891x.2004.14178.x.

7. Parkin DM, Bray F, Ferlay J, Pisani P. Global cancer statistics, 2002. CA Cancer J Clin. 2005;55:74-108. doi: 10.3322/ canjclin.55.2.74.

8. Montazeri A, Vahdaninia M, Harirchi I, Harirchi AM, Sajadian A, Khaleghi $F$, et al. Breast cancer in Iran: need for greater women awareness of warning signs and effective screening methods. Asia Pac Fam Med. 2008;7:6. doi: 10.1186/1447056X-7-6.

9. Harirchi I, Ebrahimi M, Zamani N, Jarvandi S, Montazeri A. Breast cancer in Iran: a review of 903 case records. Public Health. 2000;114:143-5. doi: 10.1038/sj.ph.1900623.

10. Mousavi SM, Montazeri A, Mohagheghi MA, Jarrahi AM, Harirchi I, Najafi M, Ebrahimi M. Breast cancer in Iran: an epidemiological review. Breast J. 2007;13:383-91. doi: 10.1111/j.1524-4741.2007.00446.x.

11. Pal SK, Mittal B. Improving cancer care in India: prospects and challenges. Asian Pac J Cancer Prev. 2004;5:226-8.

12. Chew BH, Taher SW. Primary Care for Cancers: Epidemiology, Causes, Prevention and Classification-A Narrative Review. J Family Med Community Health. 2014:1;1002.

13. Lin HP. Changing prognosis of childhood cancer--an overview. Ann Acad Med Singap. 1993;22:182-6.
14. Bredemeyer M. ACS Releases Guideline on Breast Cancer Screening. Am Fam Physician. 2016;93:711-2.

15. Tria Tirona M. Breast cancer screening update. Am Fam Physician. 2013;87:274-8.

16. Steiner E, Klubert D, Knutson D. Assessing breast cancer risk in women. Am Fam Physician. 2008;78:1361-6.

17. Tyler CV Jr, Snyder CW. Cancer risk assessment: examining the family physician's role. J Am Board Fam Med. 2006;19:46877. doi: 10.3122/jabfm.19.5.468.

18. Rao SS, Singh M, Parkar M, Sugumaran R. Health maintenance for postmenopausal women. Am Fam Physician. 2008;78:58391.

19. Chukmaitov A, Wan TT, Menachemi N, Cashin C. Breast cancer knowledge and attitudes toward mammography as predictors of breast cancer preventive behavior in Kazakh, Korean, and Russian women in Kazakhstan. Int J Public Health. 2008;53:123-30. doi: 10.1007/s00038-008-7001-9.

20. Zheng HY, Chen Q, Ye YB, Huang WW. [mRNA expression of CK19 and CEA in peripheral blood of patients with breast cancer detected by real-time quantitative PCR]. Zhonghua Yi Xue Za Zhi. 2008;88:2267-70. [Chinese].

21. Amir E, Freedman OC, Seruga B, Evans DG. Assessing women at high risk of breast cancer: a review of risk assessment models. J Natl Cancer Inst. 2010;102:680-91. doi: 10.1093/ jnci/djq088.

22. Hauk L. American college of obstetricians and gynecologists updates breast cancer screening guidelines. Am Fam Physician. 2012;85:654.

23. Chang KL, Brown L. Screening for hereditary cancer syndromes. Am Fam Physician. 2015;91(2):130-1.

24. Kaplan CP, Haas JS, Pérez-Stable EJ, Des Jarlais G, Gregorich $\mathrm{SE}$. Factors affecting breast cancer risk reduction practices among California physicians. Prev Med. 2005;41:7-15. doi: 10.1016/j.ypmed.2004.09.041.

25. Poole B, Black C, Gelmon K, Kan L. Is Canadian women's breast cancer screening behaviour associated with having a family doctor? Can Fam Physician. 2010;56:e150-7.

26. Grunfeld E, Levine MN, Julian JA, Coyle D, Szechtman B, Mirsky D, et al. Randomized trial of long-term follow-up for early-stage breast cancer: a comparison of family physician versus specialist care. J Clin Oncol. 2006;24:848-55. doi: 10.1200/JCO.2005.03.2235.

27. Maly RC, Liu Y, Diamant AL, Thind A. The impact of primary care physicians on follow-up care of underserved breast cancer survivors. J Am Board Fam Med. 2013;26:628-36. doi: 10.3122/jabfm.2013.06.120345.

28. Black JF, Meier RH. Cancer and Rehabilitation. 2018. Available at: https://emedicine.medscape.com/article/320261-overview.

29. Norman A, Sisler J, Hack T, Harlos M. Family physicians and cancer care. Palliative care patients' perspectives. Can Fam Physician. 2001;47:2009-12.

30. Kate M. O'Rourke. Improving Palliative Care for Patients with Metastatic Breast Cancer. Available at: https://www.medscape. com/viewarticle/913957.

31. Rebbeca McAteer, Caroline Wellbery, Palliative Care: Benefits, Barriers, and Best Practices. [August 4, 2021]; Available at: https://www.aafp.org/afp/2013/1215/afp20131215p807.pdf. 